\title{
La atención del paciente de traumatología y ortopedia pediátrica durante la pandemia de COVID-19
}

\author{
Pediatric Orthopedic and Trauma Care during the COVID-19 pandemic \\ Erika lliana Arana Hernández, ${ }^{*}$ Douglas Rodolfo Colmenares Bonilla, ${ }^{\ddagger}$ \\ Carlos Cuevas De Alba, ${ }^{\S}$ Héctor Hugo Flores Navarroll
}

\begin{abstract}
* Médico adscrito al Servicio de Cirugía Ortopédica Pediátrica del Benemérito Hospital Civil de Guadalajara Fray Antonio Alcalde. Encargada de la Clínica Ponseti para el tratamiento del Pie Equino Varo. ${ }^{\ddagger}$ Jefe del Servicio de Ortopedia Pediatría en el Hospital de Alta Especialidad del Bajío. Presidente de la Sociedad Mexicana de Ortopedia y Traumatología Pediátrica A.C. \& Encargado del Servicio de Cirugía Ortopédica Pediátrica del Benemérito Hospital Civil de Guadalajara Fray Antonio Alcalde. " Médico adscrito al Servicio de Cirugía Ortopédica Pediátrica del Benemérito Hospital Civil de Guadalajara Fray Antonio Alcalde. Encargado de la Clínica de Osteogénesis Imperfecta.
\end{abstract}

Correspondencia: Dra. Erika lliana Arana Hernández. Av. La Paz No. 2475, Col. Arcos Sur, 44150, Guadalajara, Jalisco, México. Correo electrónico: draeri15@hotmail.com

\section{RESUMEN}

Se establecen las recomendaciones de la Federación Mexicana de Colegios de Ortopedia y Traumatología, A.C. específicas para la Subespecialidad en Ortopedia y Traumatología Pediátrica. Se mencionan los diferentes escenarios de la práctica del ortopedista pediatra en sus actividades asistenciales médicas, quirúrgicas, de urgencia, electivas y administrativas. Estos cuidados y prerrogativas varían completamente en cada área, y es necesario establecerlas para evitar la subutilización o sobreutilización de recursos de protección. Se extienden recomendaciones en cuatro apartados particulares definidos como protección al médico, protección al personal paramédico, protección al paciente y familiares así como la seguridad del médico en actividades no asistenciales como educación continua y trabajo administrativo. Asimismo, se exponen las diferentes alternativas de uso de consultas y actividades asistenciales, al igual que de educación y educación médica continua en las que se incluyen, además de la telemedicina, las diferentes plataformas y herramientas para que, utilizando la forma remota, el médico

\section{ABSTRACT}

The recommendations of the Federación Mexicana de Colegios de Ortopedia y Traumatología A.C. are established, specific for the Subespeciality in Pediatric Orthopaedics and Traumatology. The different scenarios of the pediatric orthopaedics actions in medical, surgical, emergency, elective and administrative activities are mentioned. These cares and prerogatives vary completely in each area, and it is necessary to establish them to avoid the under or overuse of protection resources. Recommendations are made in 4 specific sections defined as: Protection of the Physician, protection of paramedical personnel, protection of the patients and relatives, as well as the safety of the physician in non-healthcare activities such as continuing education and administrative work. Likewise, the different alternatives for the use of consultations and assistance activities, as well as continuing medical education, wich include, in addition to telemedicine, the different platforms and tools so that, using the virtual form, the 
residente de ortopedia y traumatología y los Fellows en ortopedia pediátrica no interrumpan sus consultas, cursos y -capacitaciones o sus clases en pregrado o postgrado. Por último, y no menos importante, independientemente de la especialidad, el médico tiene que contar con conocimientos básicos de la enfermedad por COVID-19 incluyendo diagnóstico y tratamiento inicial, cuadro clínico, periodo de contagio, comorbilidades, etcétera.

Palabras clave: Ortopedia pediátrica, traumatología pediátrica, fracturas en niños, COVID-19, pandemia. resident in Orthopaedics and Traumatology and Pediatric Orthopaedic fellowship, do not interrupt their consultations, courses and trainings or classes in pre or post grade. Last but not least, regardless of speciality, the physician must have basic knowledge of COVID-19 disease including initial diagnosis and treatment, clinical symptoms, contagion period, comorbidities, etcetera.

Keywords: Pediatric orthopaedics, pediatric trauma, fractures in children, COVID-19, pandemic.

\section{POSTURAS Y RECOMENDACIONES}

Todas las recomendaciones van encaminadas a la atención de urgencias ortopédicas de los pacientes pediátricos durante la pandemia, la promoción del uso de equipo de protección adecuado y el cuidado de los recursos de salud. ${ }^{1}$

Se proponen las siguientes recomendaciones generales en la ortopedia y traumatología pediátrica:

1. Limitar las cirugías y acortar las estancias hospitalarias.

2. Limitar las consultas electivas y sólo atender urgencias.

3. Limitar tratamientos prolongados como yesos seriados.

Lo anterior se propone tras los siguientes razonamientos:

- Quienes atendemos niños estamos en riesgo, particularmente porque éstos no siguen indicaciones, gritan, tosen, estornudan, lloran y nos tocan sin previo aviso. Con todo ello, emiten gotitas de Flügge y aerosoles respiratorios sujetos de contaminación. ${ }^{2}$

- Debemos cuidar los recursos materiales y humanos para atender la pandemia, especialmente en un medio institucional, pero no sólo en éste.

- Los anestesiólogos también están en alto riesgo y pueden ser personal muy valioso para atender pacientes graves en caso de saturación de los servicios de salud. Debemos cuidarlos.

- Los pacientes que acuden a los servicios de salud se suman a un riesgo innecesario con implicaciones para su vida, salud ${ }^{3}$ e incluso legales. Aunque la evidencia en la población pediátrica es muy limitada, en adultos, pacientes portadores en cirugía electiva tienen hasta $20 \%$ de mortalidad al desarrollar la enfermedad. ${ }^{4}$

Se proponen las siguientes recomendaciones específicas con relación a los diferentes escenarios de la práctica del ortopedista pediatra en sus actividades asistenciales médicas y quirúrgicas, de urgencia y electivas: 


\section{En la consulta externa}

1. Dividir las citas en programables y urgentes. ${ }^{5}$

2. Atender solamente pacientes que requieran una cita urgente o algún procedimiento como retiro de yesos circulares, retiro de clavillos, revisiones o curaciones de heridas amplias o contaminadas.

3. Evitar visitas para retiro de puntos de sutura. Usar suturas bioabsorbibles para cierre de piel.

4. Utilizar férulas ${ }^{6}$ en lugar de yesos circulares para que puedan hacer el retiro en casa según el tiempo indicado, apoyados por videollamadas, mensajes, imágenes, telemedicina o comunicación por correo electrónico. Incluso implementar una clínica virtual de fracturas. ${ }^{6}$

5. Si es factible, dar atención de pacientes con displasia de cadera durante su tratamiento con arnés de Pavlik (menores de seis meses de edad). Si la fase de contagio es muy avanzada y los padres cooperan, se recomienda dar seguimiento por telemedicina o videollamada. ${ }^{7}$

\section{En urgencias pediátricas}

1. Tratar de salir del servicio de urgencias lo antes posible debido a que allí llegan de la calle los pacientes con sospecha de COVID-19.

2. Realizar en el área de cirugía ambulatoria o sala choque todos los procedimientos que sea posible para disminuir admisiones hospitalarias (reducciones cerradas, inmovilización con férulas).

3. Siempre considerar el tratamiento no quirúrgico (cuando sea una opción viable).

4. Considerar aceptar angulaciones residuales con capacidad de remodelación, especialmente en clavícula, radio distal, húmero proximal y cuello radial. Dar seguimiento con consultas más espaciadas, (por ejemplo, cada cuatro semanas). ${ }^{8}$

5. Considerar admisión hospitalaria en los siguientes eventos patológicos: artritis séptica, osteomielitis y/o absceso subperióstico, sospecha de trauma no accidental, fracturas expuestas, fracturas o lesiones de columna y pelvis, politrauma, fracturas femorales en pacientes mayores de seis años, fracturas con desplazamiento articular o periarticular como las supracondíleas humerales y femorales; igualmente en cualquier lesión fisaria de fémur y tibia.

\section{En el área de hospitalización}

Tratar de resolver las necesidades de pacientes hospitalizados como cirugías de forma inmediata. Evitar estancias hospitalarias prolongadas. Agilizar las altas hospitalarias el mismo día de la intervención quirúrgica en los casos que sea posible. ${ }^{5}$ En ciertas circunstancias considerar amputación sobre la reconstrucción en fracturas expuestas de acuerdo con la escala MESS que comprometen la viabilidad de músculos, tendones, ligamentos y huesos, evitando el prolongado internamiento. 


\section{Las cirugías electivas}

Los pacientes para quienes la cirugía se considera electiva son aquéllos con problemas crónicos cuya cirugía ciertamente puede retrasarse sin un daño significativo para el paciente o el resultado final. ${ }^{9}$ Dichas cirugías incluyen: retiros de implante, osteotomías por displasia de cadera en adolescentes, corrección de deformidades angulares, caderas luxadas por arriba de los 12 meses, tenotomías y/o alargamientos músculo-tendinosos en pacientes con parálisis cerebral, corrección de deformidades de extremidades superiores y/o inferiores, artropatía hemofílica, tratamiento quirúrgico de la escoliosis, etcétera.

\section{Cirugías consideradas urgencias relativas que pudieran ser electivas}

A medida que avanzan los contagios por el virus, se puede considerar la cirugía ambulatoria de acuerdo con la disponibilidad de recursos. ${ }^{9}$ Tal es el caso de los pacientes con displasia de cadera menores de 12 meses, ${ }^{7}$ deslizamiento de la cabeza femoral, reducción de cadera paralítica, biopsias de tumores claramente benignos, corrección de mala unión, tratamiento quirúrgico de la osteocondritis disecante, etcétera. Si es posible, estos procedimientos deben ser ambulatorios para minimizar el uso de recursos.

\section{Urgencias quirúrgicas}

A medida que avanzan los contagios por el virus, la optimización de recursos cobrará mayor importancia y la realización de cirugías se volverá más limitada. Bajo condiciones de urgencia se deben considerar las lesiones en las que la intervención quirúrgica inmediata evitaría un deterioro significativo de la función, ${ }^{9}$ como osteosíntesis de fracturas de fémur, tibia, reducción y fijación de fracturas supracondíleas humerales, cóndilo humeral, fracturas de antebrazo en adolescentes, biopsia de tumor aparentemente maligno, amputaciones de extremidades necróticas, etcétera.

\section{Emergencias quirúrgicas}

En caso de saturación de hospitales y las UCls, la escasez de recursos es crítica. En esta situación, sólo las verdaderas lesiones que amenazan la vida - las extremidades deben llevarse a cirugía con el objetivo de minimizar la necesidad eventual de asistencia con el ventilador. ${ }^{9}$ Algunas condiciones que no se pueden diferir son: politrauma, sepsis por osteomielitis, artritis séptica, absceso subperióstico, fracturas expuestas Gustilo II y III, síndrome compartimental, luxaciones agudas, fracturas con compromiso vascular, amputaciones traumáticas. 
Recomendaciones de protección al personal, pacientes y familiares:

\section{Protección al ortopedista}

\section{En consultorio:}

1. Espaciar las citas hasta cada 45 minutos por paciente.

2. Solicitar anticipadamente que acuda un solo acompañante por paciente.

3. Enfatizar en la puntualidad de inicio y terminación de la consulta.

4. Hacer «triage o cribado» en la selección de los pacientes que solicitan consulta.

5. Generalizar uso de ropa y textiles de consultorio desechables o limpios con cambio entre cada paciente.

6. Uso de anteojos que cubran lateralmente o goggles.

7. Uso de cubrebocas quirúrgicos.

8. Uso de guantes en caso de secreciones, heridas, fluidos corporales.

9. Lavado de manos con agua y jabón según los cinco momentos para el lavado de manos. Secado con toallas desechables al inicio y término de cada consulta.

10. Sanitizar o higienizar asientos, picaportes y sitios de contacto habitual con soluciones alcoholadas o cloradas (las últimas en dilución al 0.05-0.1\%; recordar que permanecen activas sólo 24 horas).

11. Dimensionar y mantener la sana distancia.

\section{En hospital en áreas de bajo riesgo (consulta externa, áreas} administrativas, recursos humanos, etcétera)

1. Aseo de manos con jabón (según los cinco momentos para el lavado de manos) y/o alcohol en gel al entrar y salir, mantener puerta cerrada en todo momento.

2. Uso continuo de cubrebocas quirúrgico.

3. Uso de anteojos que cubran lateralmente o goggles.

4. Uso del equipo diagnóstico (ej. martillo de exploración, goniómetros, cinta métrica, etcétera) sin sacarlo de esa área.

5. Desinfección constante de plumas, lápices y otros instrumentos que pudieran actuar como fómites.

En hospital en áreas de riesgo intermedio (quirófano, urgencias pediatría, urgencias de adultos, tococirugía)

1. Aseo de manos con jabón (según los cinco momentos para el lavado de manos) y/o alcohol en gel al entrar y salir, mantener puertas cerradas en todo momento.

2. Uso de mascarilla N95 o equivalente.

3. Uso de anteojos que cubran lateralmente o goggles y/o careta.

4. Uso de guantes de látex y bata desechables para cada paciente.

5. Instrumental y equipo propio del área. 
En hospital en áreas de alto riesgo (unidad de cuidados intensivos, áreas COVID, cualquier área con pacientes sospechosos o positivos a COVID-19) ${ }^{1}$

1. Uso de mascarilla N95, FPP2 o FPP3.

2. Uso de anteojos que cubran lateralmente o goggles y careta.

3. Batas y botas impermeables desechables, gorros o pasamontañas desechables.

4. Guantes de nitrilo.

A pesar de que las guías recomiendan uso de cubrebocas convencionales al estar en zonas con bajo riesgo de contagio en hospitales y consultorios, Xiaodong Guo y colaboradores recomiendan enfáticamente el uso de mascarillas N95 durante todo el tiempo y en cualquier área del hospital donde el ortopedista realice labores asistenciales y concluyen que la falta de uso de este tipo de mascarillas está relacionada con alto riesgo de contagio con COVID-19 con un odds ratio de $5.2 .^{3}$

\section{Protección a personal paramédico}

1. Lavado continuo de manos con agua y jabón (durante al menos 20 segundos) $)^{5}$ y/o uso de alcohol en gel.

2. Uso de cubrebocas convencional.

3. Limpiar de manera rutinaria y constante las superficies y objetos que se tocan con frecuencia en su área de trabajo. ${ }^{10}$

4. Mantener distancia mayor de 1.5 a 2 metros con los pacientes.

5. Al toser o estornudar, cubrirse con un pañuelo desechable y tirarlo a la basura (posterior a esto, lavarse las manos) $^{10}$

6. El personal de recepción debe proporcionar, al confirmar las citas, información respecto a cuidados de los pacientes y familiares antes, durante y después de su consulta.

7. Toma de temperatura corporal con termómetros digitales a distancia.

8. Evitar saturación de la sala de espera con la conservación de la sana distancia.

9. Sanitizar o higienizar asientos, picaportes y sitios de contacto habitual con soluciones alcoholadas, cloradas.

10. Uso de tapetes sanitizantes.

11. Adaptación de protección a los escritorios de las asistentes (mampara de acrílico transparente).

\section{Protección a pacientes y familiares}

1. El paciente debe acudir a consulta con un solo familiar o tutor.

2. Uso de cubrebocas a tiempo completo (paciente y acompañante).

3. Lavarse las manos al llegar al consultorio, (por al menos 20 segundos) y/o uso de alcohol en gel.

4. Deben acudir a consulta directamente de casa, sin haberse expuesto a lugares públicos. 
5. Si presentan síntomas respiratorios (tos, estornudos, dolor de garganta, fiebre, malestar general) ya sea el paciente o su acompañante, deberán reprogramar la cita.

6. Mantener distancia al momento del interrogatorio.

\section{Seguridad del médico en actividades no asistenciales}

Continuar con la educación del ortopedista en formación es fundamental. Se recomienda el uso de plataformas y herramientas tecnológicas para que, utilizados de manera remota o virtual, los residentes en formación no interrumpan sus cursos, capacitaciones y/o clases de pregrado o postgrado. Con el uso de estas plataformas evitan someterse al riesgo que implica estar en un lugar conglomerado de personas.

También se recomienda que durante las actividades administrativas que se realicen en la oficina se tomen medidas de sanitizar o higienizar asientos, picaportes, apagadores de luz y sitios de contacto habitual con soluciones alcoholadas, cloradas así como mantener la sana distancia.

\section{CONCLUSIONES}

Debido a la pandemia por COVID-19 las recomendaciones para la atención de pacientes pediátricos se resumen en limitar la consulta, tratamientos y cirugías electivas, proporcionar la atención de urgencias, el uso de protección adecuada de los ortopedistas, personal paramédico, pacientes y familiares y el cuidado de los recursos de salud.

\section{BIBLIOGRAFÍA}

1. Zhen CL, Wilson W, Diarmuid M, James H. The Orthopaedic forum. Novel coronavirus and orthopaedic surgery. J Bone Joint Surg. 2020; 102 (9): 734-744. Available in: https://www.jbjs.org/reader. php?id=106928\&rsuite_id=2371125\&native=1\&source=The_Journal_of_Bone_and_Joint_Surgery\% 2F102\%2F9\%2F745\%2Ffulltext\#info.

2. Murillo-Godínez G. Las gotitas de Flügge. Rev Med Inst Mex Seguro Soc. 2009; 47 (3): 290. Available in: https://www.medigraphic.com/pdfs/imss/im-2009/im093j.pdf.

3. Xiaodong G, Jiedong W, Dong H, Lisha W, Li G, Yang W, et al. The orthopaedic forum. survey of COVID-19 disease among orthopaedic surgeons in Wuhan, People's Republic of China. J Bone Joint Surg Am. 2020; 102 (10): 847-854. Available in: https://www.jbjs.org/reader. php?id=107030\&rsuite_id=2391045\&native=1\&source=The_Journal_of_Bone_and_Joint_ Surgery/102/10/847/fulltext\&topics=if\#info

4. Lei S, Jiang F, Su W, Chen C, Chen J, Mei W, et al. Clinical characteristics and outcomes of patients undergoing surgeries during the incubation period of COVID-19 infection. E Clinical Medicine. 2020; 21 (100331). Available in: https://www.thelancet.com/action/showPdf?pii=S2589-5370\%2820\%2930075-4.

5. Sociedad Española de cirugía ortopédica y traumatología. Recomendaciones generales de la Sociedad Española de Cirugía Ortopédica y Traumatología frente al Covid-19. 2020. Disponible en https:// www.secot.es/media/docs/covid19/RecomendacionesSECOTGeneralesDeCOTFrenteAICovid19. pdf

6. NHS. Clinical guide for the management of trauma and orthopaedic patients during the coronavirus pandemic. 2020. Available in: https://www.england.nhs.uk/coronavirus/wp-content/uploads/ sites/52/2020/03/C0274-Specialty-guide-Orthopaedic-trauma-v2-14-April.pdf.

7. Castañeda PG, Price CT, Sankar WN, Upasani VS, Kasser JR, Mubarak SJ, et al. Suggestions for managing DDH in a resource limited environment during the COVID-19 pandemic. International Hip 
Displasia Institute. 2020. Available in: https://hipdysplasia.org/?s=Suggestions+for+managing+DDH+i $\mathrm{n}+\mathrm{a}+$ resource+limited+environment+during+the+Covid-19+pandemic.

8. Fracture care guidelines in the time of COVID19 for CMC UTSW. Pediatric Orthopaedic Society of North America, Blogs April 2020. Available in: https://posna.org/Blogs/POSNA-COVID-19-Updates/April-2020/ Fracture-Care-in-the-Time-of-COVID19.

9. Guy DK, Bosco JA, Savoie FH. AAOS Guidelines for elective surgery during the Covid-19 pandemic. April 2020. Available in: https://www.aaos.org/about/covid-19-information-for-our-members/aaos-guidelinesfor-elective-surgery/.

10. Zhou W, Zhong N, Zhu S. The coronavirus prevention handbook, 101 scince-based tips that could save your life. 2020. Available in: https://g.co/kgs/h4mM72. 